\title{
Mesa-height Dependent Quantum Efficiency Characteristics of InGaN Micro-LEDs
}

\author{
Chao Shen, Tien Khee Ng, Chun Hong Kang and Boon S. Ooi \\ Photonics Laboratory, King Abdullah University of Science and Technology (KAUST), Thuwal 23955-6900, Kingdom of Saudi Arabia \\ *email: boon.ooi@kaust.edu.sa
}

\begin{abstract}
The mechanisms of mesa-height dependent efficiency and efficiency droop of blue $\mathrm{InGaN} / \mathrm{GaN}$ micro-LED is presented. Device with a large etch-depth $(>1.3 \mu \mathrm{m})$ shows significant strain relief with aggravated current crowding.

OCIS codes: (230.3670) Light-emitting diodes; (250.5590) Quantum-well, -wire and -dot devices
\end{abstract}

\section{Introduction}

InGaN-QW-based micro-light-emitting diode (micro-LED) structure was demonstrated to hold unique electrical characteristics suitable for high power LED lighting compared with its large-area counterparts [1-4]. Micro-LEDs show improved efficiency at high injection current density although the efficiency was affected via side-wall defects induced non-radiative recombination at low injection [4]. The high efficiency at high injection was attributed to enhanced current spreading in micro-LEDs, which resulted in a reduction of efficiency droop effect [5]. Other potential advantages of micro-LED include enhanced light extraction [6] and suitability in optoelectronic integration [7]. To realize high efficiency micro-LED, the size effect was studied experimentally and by simulation [3, 4]. However, further exploration on the design of mesa height $(H)$ is required. Our previous research work suggested that the internal quantum efficiency in micro-LED was improved due to the enhanced strain relief in micro-LEDs with a large etch depth [8]. Thus the investigation of mesa height related to the quantum efficiency characteristics of micro-LEDs is crucial.

The objective of this research is to look into the peak efficiency and efficiency droop at high injection current level from micro-LEDs with different mesa height. Our analysis revealed increased peak efficiency in micro-LED with a large $H$, which was attributed to residual strain relief effect. Besides, an aggravation of efficiency droop was observed owing to an extra vertical current transport process.

\section{Experiments}

The InGaN/GaN LED is in a p-i-n configuration grown on c-plane sapphire substrate with 12 -stack $3 \mathrm{~nm}$-InGaNwell / 10nm-GaN-barrier multiple quantum wells (MQWs) as the active layer (see Fig. 1 (a)). The LED structure is characterized using transmission electron microscopy (TEM) shown in Fig. 1(b). The micro-LEDs with various mesa heights $(H=0.5 \sim 4 \mu \mathrm{m})$ were fabricated using UV contact-lithography and $\mathrm{Cl}_{2}$ based inductively-coupled plasma (ICP) etching. The diameter of the circular devices was fixed at $30 \mu \mathrm{m}$. The fabricated micro-LEDs were tested in a home-made LED probe station integrated with a microscope, Keithley 2400 source meter and Newport 2936C power meter. Micro-Raman spectra were measured using a $532 \mathrm{~nm}$ laser at room temperature.

(a)

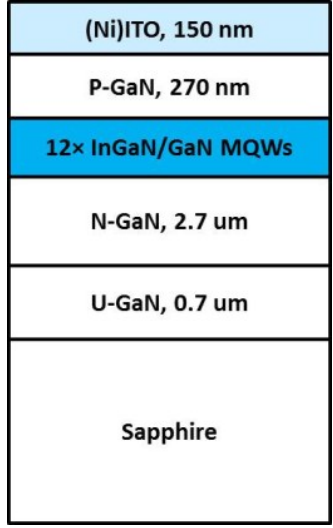

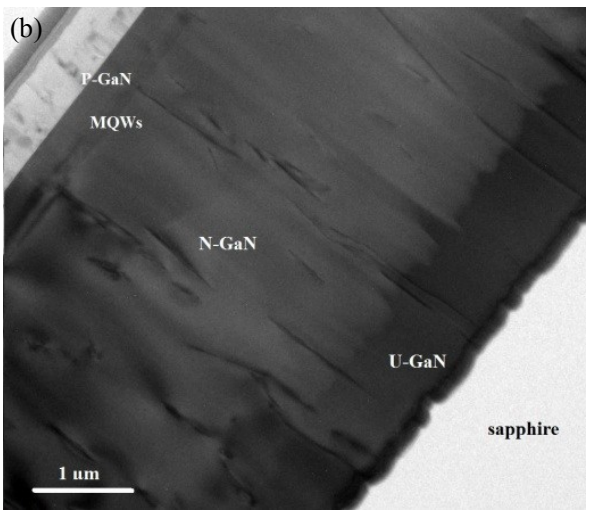

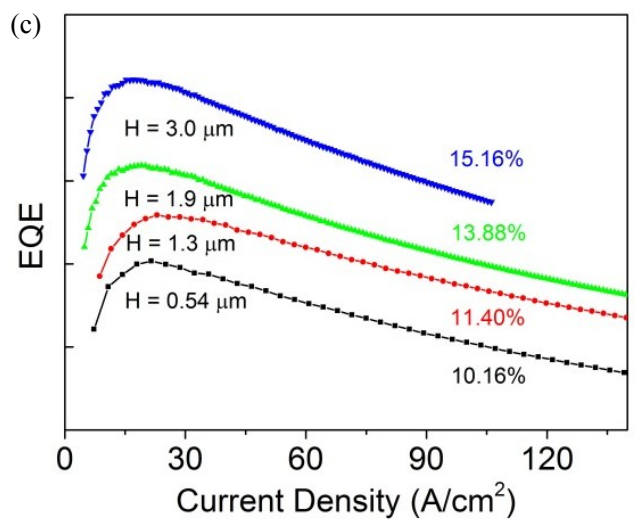

Fig.1 (a) Schematic drawing of LED structure with sputtered Ni-ITO p-contact. (b) TEM cross-sectional image of InGaN/GaN LED epitaxy layer structure. (c) Relative external quantum efficiency (EQE) vs. injection current density in micro-LEDs with various $H$. The EQE droop at 100 $\mathrm{A} / \mathrm{cm}^{2}$ of each micro-LED is indicated.

\section{Results and Discussion}


Figure 1(c) shows the external quantum efficiency (EQE) vs. MESA height characteristics obtained from $L-I$ measurements. The efficiency droop is evident in all the devices, where a progressive increase in the peak EQE $\left(\eta_{\text {peak }}\right)$ with increasing $H$ is observed. With increasing current injection, the EQE steadily drops beyond $\eta_{\text {peak }}$. Comparing the EQE droop at $100 \mathrm{~A} / \mathrm{cm}^{2}\left[\left(\eta_{\text {peak }}-\eta_{200}\right) / \eta_{\text {peak }}\right]$, we observed an aggravation of efficiency droop in micro-LEDs with larger $H$. The reasons for such observation are explored further in the following.

There exists the polarization fields in the III-N materials due to both non-centro-symmetric GaN unit-cell related spontaneous polarization field and strain related piezoelectric polarization field. The latter arises from the lattice and thermal mismatches between epitaxial layers with the substrate. The strain field is expected to be partly relieved in LED micro-structures, and hence improving the electron-hole wavefunction overlapping, leading to the efficiency improvement. In order to probe the strain relief in micro-LEDs, micro-Raman spectra were collected from the center of each MESA as shown in Fig. 2(a). The Raman peak positions of $E_{2}$ and $A_{1}$ optical phonon modes represent the strain level in GaN top layers, which is directly related to the strain level in active layer [9]. The apparent wavenumbers down-shift (i.e. shifted to lower wavenumber) for $\mathrm{E}_{2}$ mode was measured with increasing $H$, indicating a significant strain relief. Taking the strain-free $\mathrm{E}_{2}$ wavenumber to be $567.25 \mathrm{~cm}^{-1}$, the in-plane strain in $\mathrm{GaN}$ thin film was reduced from $-1.5 \mathrm{GPa}$ (corresponding to $570.8 \mathrm{~cm}^{-1}$ in micro-LED with $H=0.54 \mu \mathrm{m}$ ) to -0.8 $\mathrm{GPa}$ (corresponding to $569.3 \mathrm{~cm}^{-1}$ in micro-LED with $H=3.0 \mu \mathrm{m}$ ) based on the crystal-symmetry related formalism [8]. Therefore an enhanced strain relief in InGaN MQWs was deduced in micro-LEDs with increasing mesa height, leading to the increased peak efficiency.

Growing on insulating sapphire substrate, InGaN/GaN LED epitaxy also suffers from the effect of non-uniform current distribution, or the current crowding effect, resulting in an exacerbated efficiency droop [5]. The current spreading in an InGaN/GaN micro-LED is illustrated in Fig. 2(b). For micro-LEDs with increasing mesa height, the vertical current transport component has to be considered in addition to the lateral transport component. With both current transport processes, the growing current crowding effect in micro-LEDs with increasing $H$ led to the aggravated efficiency droop.
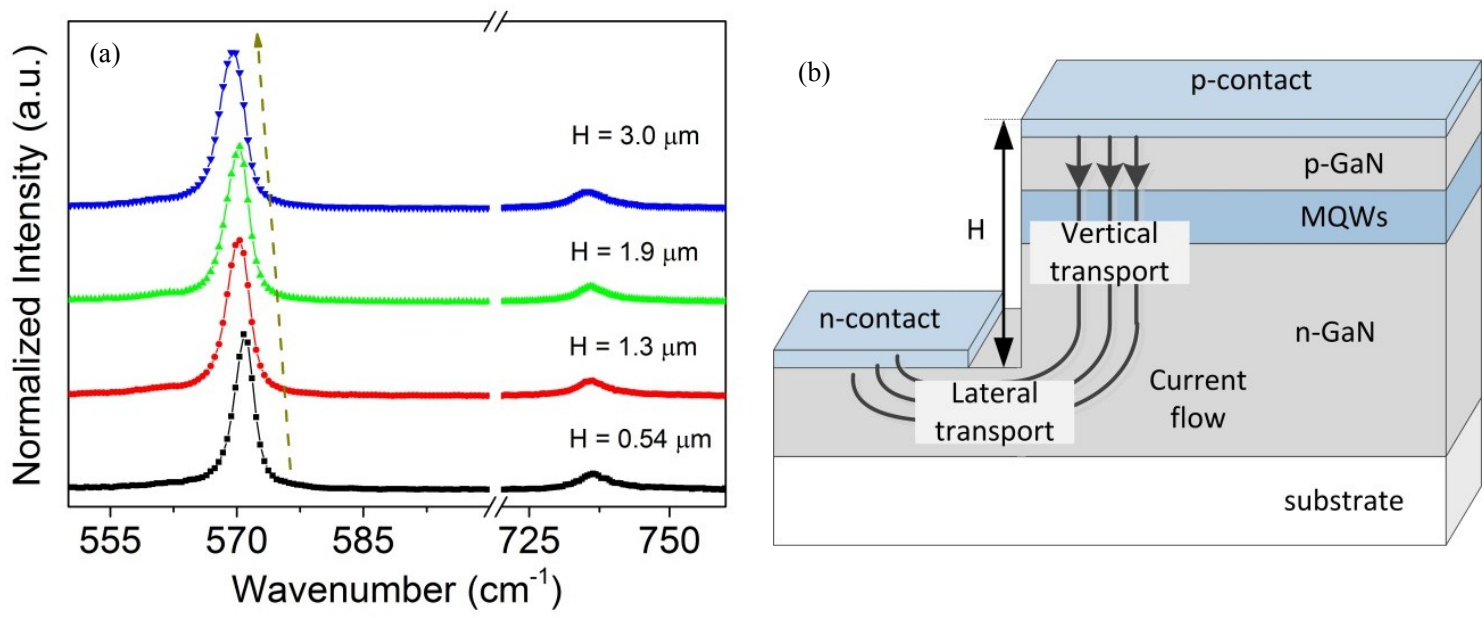

Fig.2 (a) Raman spectra collected from the center of each micro-LED. (b) Illustration of current spreading model in micro-LEDs with a considerable etch depth $(H)$.

\section{Conclusions}

In conclusion, we have investigated the mesa height dependent efficiency and efficiency droop in InGaN/GaN micro-LEDs. With a larger mesa height, the micro-LED shows increasing peak efficiency owing to an enhanced strain relief albeit with a drawback of aggravated efficiency droop due to current crowding effect. The results indicate that the optimization of strain relief and current spreading are required when designing micro-LEDs for high brightness lighting applications.

\section{References}

[1]A. E. Kelly, J. J. D. McKendry, S. Zhang, D. Massoubre, B. R. Rae, R. P. Green, et al., "High-speed GaN micro-LED arrays for data communications," in Transparent Optical Networks (ICTON), 2012 14th International Conference on, 2012 , pp. 1-5.

[2]Z. J. Liu, K. M. Wong, C. W. Tang, and K. M. Lau, "GaN-based monolithic LED micro-arrays," in OptoElectronics and Communications Conference, 2009. OECC 2009. 14th, 2009, pp. 1-2.

[3]Y. B. Tao, S. Y. Wang, Z. Z. Chen, Z. Gong, E. Y. Xie, Y. J. Chen, et al., "Size effect on efficiency droop of blue light emitting diode," Physica Status Solidi C: Current Topics in Solid State Physics, No 3-4, vol. 9, pp. 616-619, 2012. 
[4]P. F. Tian, J. J. D. McKendry, Z. Gong, B. Guilhabert, I. M. Watson, E. D. Gu, et al., "Size-dependent efficiency and efficiency droop of blue InGaN micro-light emitting diodes," Applied Physics Letters, vol. 101, Dec 32012.

[5]V. K. Malyutenko, S. S. Bolgov, and A. D. Podoltsev, "Current crowding effect on the ideality factor and efficiency droop in blue lateral InGaN/GaN light emitting diodes," Applied Physics Letters, vol. 97, Dec 202010.

[6]J. H. Zhu, L. J. Wang, S. M. Zhang, H. Wang, D. G. Zhao, J. J. Zhu, et al., "Light extraction efficiency improvement and strain relaxation in InGaN/GaN multiple quantum well nanopillars," Journal of Applied Physics, vol. 109, Apr 152011.

[7]Z. Shuailong, J. J. D. McKendry, G. Zheng, B. R. Rae, S. Watson, X. Enyuan, et al., "Directly color-tunable smart display based on a CMOScontrolled micro-LED array," in Photonics Conference (IPC), 2012 IEEE, 2012, pp. 435-436.

[8]C. Shen, T. K. Ng, D. Cha, and B. S. Ooi, "Strain Relief InGaN/GaN MQW Micro-pillars for High Brightness LEDs," in 2013 Saudi International Electronics, Communications and Photonics Conference (SIECPC), 2013.

[9]A. G. Kontos, Y. S. Raptis, N. T. Pelekanos, A. Georgakilas, E. Bellet-Amalric, and D. Jalabert, "Micro-Raman characterization of InxGa1xN/GaN/Al2O3 heterostructures," Physical Review B, vol. 72, p. 155336, Oct 2005. 